\title{
Fabrication and Characterization of Bragg grating in CYTOP POF at $600 \mathrm{~nm}$ wavelength
}

\author{
Rui Min ${ }^{1}$, Beatriz Ortega ${ }^{1}$, Arnaldo Leal-Junior ${ }^{2}$, Carlos Marques ${ }^{3}$ \\ ${ }^{1}$ ITEAM Research Institute, Universitat Politècnica de València, 46022 Valencia, Spain \\ ${ }^{2}$ Graduate Program of Electrical Engineering of Federal University of Espirito Santo, Vitória, Brazil \\ ${ }^{3}$ Instituto de Telecomunicações, Campus Universitário de Santiago, 3810-193 Aveiro, Portugal
}

\begin{abstract}
Received 1 xxx 201x, revised $25 x x 201 x$, accepted $x x$ xx 201x, published 5 xx 201x, current version $x x$ xxx 201x. (Dates will be inserted by IEEE; "published" is the date the accepted preprint is posted on IEEE Xplore ${ }^{\circledR}$; "current version" is the date the types et version is posted on Xplore ${ }^{\circledR}$ ).
\end{abstract}

\begin{abstract}
We demonstrate Bragg grating inscription in commercial CYTOP POF at a wavelength of $600 \mathrm{~nm}$ using a $248 \mathrm{~nm}$ krypton fluoride (KrF) excimer laser system with a repetition rate of $40 \mathrm{~Hz}$ and average pulse energy of $0.60 \pm 0.02$ $\mathrm{mJ}$ during $60 \mathrm{~min}$. Experimental characterization of the Bragg grating is performed under different conditions, where the temperature and strain sensitivities were accurately measured to define potential sensing and optical communication applications. Furthermore, different humidity conditions were experimentally tested and it was proved CYTOP POF as a good candidate for sensing applications under humidity change environment.
\end{abstract}

Index Terms_-Polymer optical fibers, fiber Bragg gratings, optical fiber devices, CYTOP, optical filters.

\section{INTRODUCTION}

With the continued development of fabrication technologies and materials over the last two decades, polymer optical fiber (POF) has attracted huge attention for sensing and communication applications, due the advantage such as low Young's modulus and biocompatibility which make POF suitable for large strain measurements and medical sensing applications. POFs with large cores have been also shown as a cost attractive solution for short-range optical communication due to less operational complexity and simpler installation when compared with standard silica fiber [1]. Fiber Bragg gratings (FBGs) in POF exploit the advantages given by the low Young's modulus and biocompatible nature of the POF material and lead to develop a new generation of sensors. So far, most of the research is based on poly (methyl methacrylate) (PMMA) microstructured POFs (mPOFs) [2] and step index POF [3], where the mPOFs could show an endless single mode performance provided certain conditions are satisfied [4]. Such performance is difficult to achieve in PMMA step index POFs due to the higher refractive index difference between core and cladding. However, the connection between $\mathrm{mPOF}$ and silica fiber is a serious challenge due to holes from microstructure. Furthermore, some index matching gel can be employed to improve this coupling but the gel gets inside the holes, and leads to a harmful effect on the light transmission. In this way, step index PMMA is a suitable POF to solve this issue and, recently, a single mode PMMA POF with doped core for photosensitivity increase was investigated and FBGs were inscribed to be used as optical sensors for medical applications [5]. Similarly, to other POFs, high fiber loss has proven a major issue in the widespread of sensing applications. The high transmission loss in PMMA POF are due to higher overtone vibrations of $\mathrm{C}-\mathrm{H}$ groups which lead to broad-spectrum absorption with peaks in the infrared region [6].

Recent investigations on perfluorinated optical materials such as cyclic transparent fluoropolymers (CYTOP) show good transmission and overcomes this limitation. The wavelength of the maximum absorption is determined by the individual molecular structure. CYTOP only contains C-C, C-O and C-F bonds and shows higher transparency and low absorption losses due to the highly amorphous nature [6].

However, the benefit of low loss performance given by CYTOP also limits the photosensitivity of the fiber. In 2014, M. Koerdt et al [7] reported the first FBG in CYTOP fiber at $1450 \mathrm{~nm}$ spectral region, where a $248 \mathrm{~nm}$ pulsed krypton fluoride $(\mathrm{KrF})$ laser was used with an irradiation fluence of $5 \mathrm{~kJ} / \mathrm{cm}^{2}$ during less than 1 hour. The authors demonstrated that the core material of the Giga POF 50SR is photosensitive at $248 \mathrm{~nm}$ wavelength, but, unfortunately, the spectral response was not good for sensing and the sensitivity could not be measured also due to coupling issues. The reflected signal spectra of this multimode fiber showed a bandwidth of around $10 \mathrm{~nm}$ composed of several peaks corresponding to principal modes [8]. Each principal mode consists of several excited guided modes in the POF whose propagation constants are almost the same. The position of the single mode launching fiber determines the excited modes in the multimode POF.

Then, most of the attention about FBG in CYTOP POF is driven to femtosecond (fs) laser irradiation, as reported in 2015 [5]. This laser system allows the FBG inscription in any type of bulk material and optical fiber without photosensitivity [9] due to the extremely large light intensity through a small spatial region and ultra-short irradiation time. Also, the refractive index changes introduced by the fs pulses are highly localized. From the first FBG inscription in CYTOP POF with fs laser reported [5], the same group has published a considerable number of works with focus in sensing applications at $1550 \mathrm{~nm}$ 
spectral region [10-13]. Due to point-by-point or plane-by-plane method limitation, the fs pulses irradiation is limited to typical periods of FBGs, due to the spatial resolution is limited by the diffraction limit [14]. Compared with silica fiber where the standard spectral region is at $1550 \mathrm{~nm}$ wavelength, POF shows huge advantages in $650 \mathrm{~nm}$ and $850 \mathrm{~nm}$ wavelength (including the compatibility to low cost LEDs close to the visible region as white and RGB LEDs). Thus, the fabrication of FBGs in low loss CYTOP fiber at these wavelengths is important for sensing and communication applications.

In this paper, we demonstrate the first FBG irradiation with $248 \mathrm{~nm}$ UV pulses at $600 \mathrm{~nm}$ spectral region, and temperature and strain characterization tests to have been carried out to propose them as novel optical devices for visible communications and sensing applications.

\section{BRAGG GRATING FABRICATION}

\section{A. Inscription describe}

This work employed the commercial graded index multimode perfluorinated POF (GigaPOF-50SR, Chromis Fiberoptics), with core diameter of $50 \mu \mathrm{m}$, a $40 \mu \mathrm{m}$ cladding layer and an additional polyester and polycarbonate over-cladding structure to protect the fiber, resulting in a total diameter of $490 \mu \mathrm{m}$ [15]. The fiber was etched with chloroform $(\mathrm{CHCl})$ [6] during several minutes in the fume hood due to the use of such dangerous chemical product. The fiber was etched piece-by-piece to avoid any fiber twisting during etching process and reduce the POF fragility induced by the etching treatment. Then, the fiber was washed in flowing water for the removal of any residual chloroform. The final diameter of the fiber was about $90 \mu \mathrm{m}$ without the over-cladding as shown in Fig. 1.

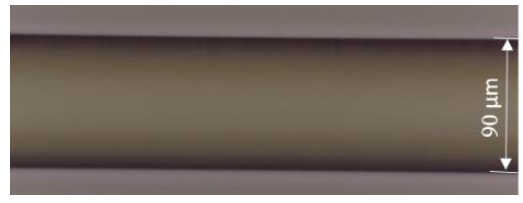

Fig. 1. The commercial graded index multimode perfluorinated fiber after etching under microscope.

One razor blade was used to cleave the fiber end and it was buttcoupled to an $8^{\circ}$ angle SM pigtail by using some index matching gel. As shown in Fig. 2, a coherent $\mathrm{KrF}$ excimer pulsed laser emitting at $248 \mathrm{~nm}$ wavelength was used. The UV beam was focused onto the fiber core utilizing a plano-convex cylindrical lens (Newport CSX200AR.10) with focal length of $200 \mathrm{~mm}$. The FBG was fabricated using the phase mask technique. The refractive index of CYTOP is about 1.34 , so a $449.05 \mathrm{~nm}$ pitch phase mask is required to fabricate FBGs in CYTOP POF at $600 \mathrm{~nm}$ wavelength region.

A $20 \mathrm{~cm}$ long CYTOP fiber was placed on the XYZ translation stage and the $248 \mathrm{~nm}$ laser pulse energy was set at $0.6 \mathrm{~mJ}$ with a repetition rate of $40 \mathrm{~Hz}$. The fiber was irradiated through the phase mask during 60 minutes. A supercontinuum laser (Fianium WL-SC400-40) was used as optical source to characterize the reflectivity response of the grating and it should be noted that, due to the high power and broad wavelength $(400 \mathrm{~nm} 2400 \mathrm{~nm})$ of the source, one filter (Semrock $595 \mathrm{~nm} 700 \mathrm{~nm}$ ) in order to protect the source in case of high power reflections.

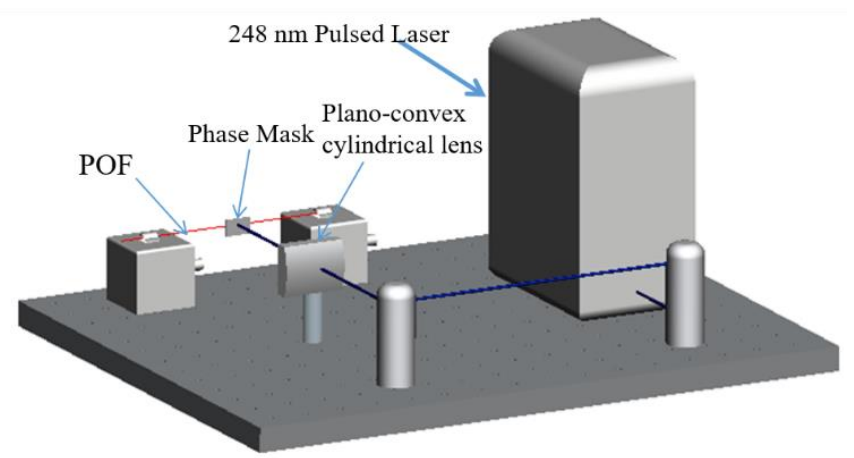

Fig. 2. Experimental setup for POFBG inscription.

\section{B. Grating performance}

An optical spectrum analyzer (Yokogawa AQ6373B) with $0.2 \mathrm{~nm}$ resolution bandwidth was connected to a SM silica pigtail and an optical circulator was employed to measure the reflected power. The length of the inscribed FBG was $10 \mathrm{~mm}$. Fig. 3 (a) shows the reflected spectrum obtained with butt-coupling method. Fig. 3 (b) shows an additional optical spectrum from the same FBG but a small change in the butt-coupling to illustrate how critical is the butt-coupling process for a good characterization of the FBG.

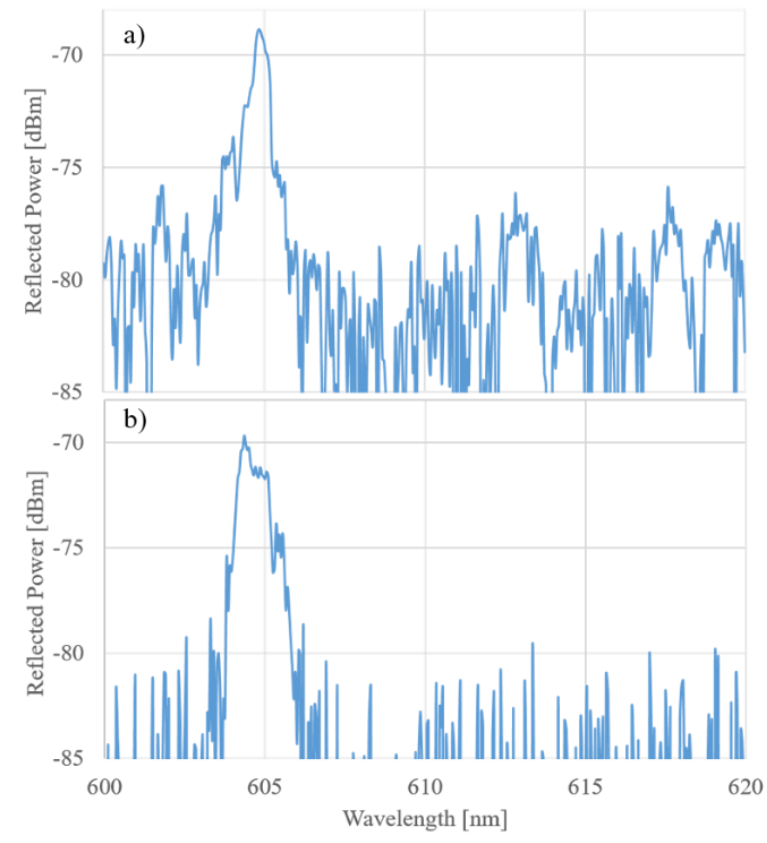

Fig. 3. Reflected power spectrum by the FBG under different butt coupling. (a) butt-coupling method. (b) intentional misalignment in the butt-coupling.

\section{TEMPERATURE AND STRAIN CHARACTERIZATION}

Temperature, humidity and strain experiment results are presented 
to assess the potential sensing and optical communication applications. one end of the POF (grating inside) was cut as a flat face with a razor blade, then it was butt coupled with an APC SM pigtail by using an UV curable adhesive (Norland 68).

The grating was placed on a Peltier plate with temperature set by an electronic temperature controller. Some silicone grease was applied on the grating section to increase the heat conduction. It was measured from $32{ }^{\circ} \mathrm{C}$ to $72{ }^{\circ} \mathrm{C}$ with $20{ }^{\circ} \mathrm{C}$ step and a temperature sensitivity of $11.2 \pm 0.5 \mathrm{pm} /{ }^{\circ} \mathrm{C}$ was obtained, providing a red shift performance, as shown in Fig.4. The results show a different behavior when compared with PMMA POF, which temperature sensitivity has been measured around $-0.069 \pm 0.001 \mathrm{~nm} /{ }^{\circ} \mathrm{C}$ [12] at $850 \mathrm{~nm}$ wavelength region. The CYTOP FBG fabrication with fs laser also showed a positive wavelength shift with increasing temperature, which can be explained in terms of the positive thermo-optic coefficient, which is close to $7.4 \times 10^{-5} / \mathrm{K}$ due to the linear expansion in bulk volume for CYTOPs [5]. Nevertheless, until now, no temperature sensitivity was reported at such wavelength.

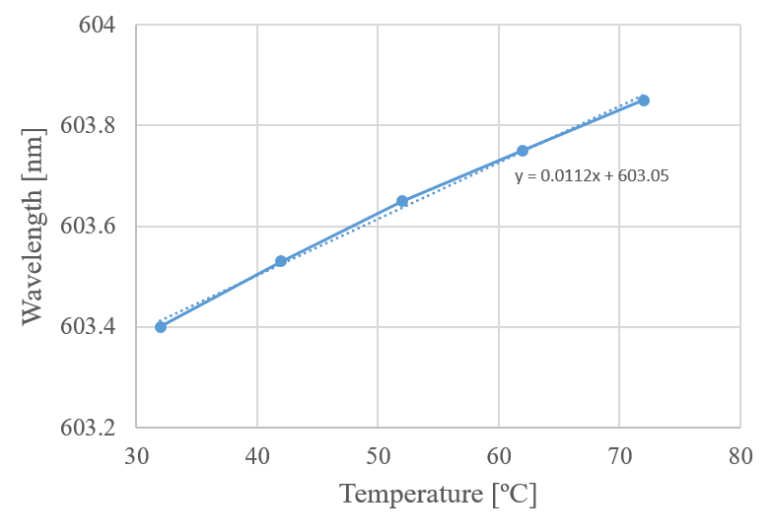

Fig. 4. Wavelength shift under different temperature.

Humidity response of the FBG was also measured and the connectorised CYTOP POF was placed in the climatic chamber (Angelantoni Industrie $\mathrm{CH} 340$ ) with constant temperature at $30{ }^{\circ} \mathrm{C}$ (a temperature chosen randomly and close to room temperature). The humidity level was from $50 \%$ to $90 \%$, and we can observe no wavelength shift for $40 \%$ humidity change as shown in Fig. 5. It indicates CYTOP POF is a good candidate for sensing applications under humidity change environment or in water.

The strain characterization was also investigated and the CYTOP POF was placed into a strain setup (see Fig. 6 (a)) for this purpose. A $13.2 \mathrm{~cm}$ long CYTOP POF including a $1 \mathrm{~cm}$ long FBG was fixed with fiber clamps (Thorlabs HF001) and a translation stage was used with $0.1 \mathrm{~mm}$ step $(0.075 \%)$ while the data was collected. The wavelength shift under different strain of the reflection spectrum is shown in Fig. 6 (b). A wavelength shift of $2.0 \mathrm{~nm}$ was obtained with respect to the unstrained grating, which indicated a linear strain sensitivity of 0.533 $\mathrm{pm} / \mu \varepsilon$ at $600 \mathrm{~nm}$ spectral region. The maximum hysteresis occurs at about $1500 \mu \varepsilon$, in which the deviation between the loading and unloading cycles is lower than $0.1 \mathrm{~nm}$.

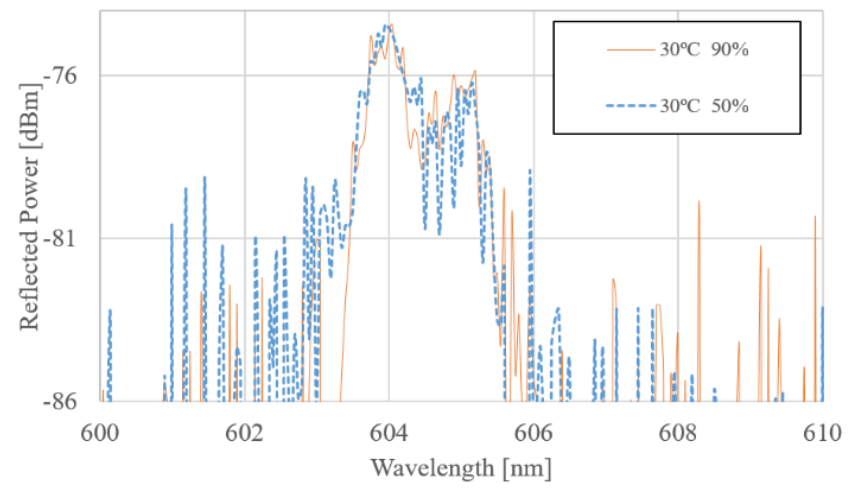

Fig. 5. FBG spectrum under different humidity level at constant temperature.

To the best of our knowledge, no reports are published in the literature on the strain sensitivity of FBGs in POF at $600 \mathrm{~nm}$ region. The strain sensitivity of PMMA mPOF Bragg grating at $850 \mathrm{~nm}$ wavelength is about $0.711 \mathrm{pm} / \mu \varepsilon$ [16] and at $1560 \mathrm{~nm}$ wavelength is around $1.58 \mathrm{pm} / \mu \varepsilon$ [17]. Nevertheless, this lower sensitivity obtained in $600 \mathrm{~nm}$ region is consistent with the FBG wavelength shift with strain that presents an upward trend with respect to the Bragg wavelength increase, since the wavelength shift $(\Delta \lambda)$ is defined as Eq. (1) for strain and temperature variations. Then, the term $(1-P e) \lambda_{B}$ is the strain sensitivity, whereas $(\alpha+\xi) \lambda_{B}$ is the temperature sensitivity. For this reason, if the Bragg wavelength $\left(\lambda_{B}\right)$ is in shorter wavelengths, the strain and temperature sensitivities will be lower when comparing with the one of $\lambda_{B}$ in longer wavelength region, such as $850 \mathrm{~nm}$ or $1550 \mathrm{~nm}[12]$.

$$
\Delta \lambda_{B}=\left[\left(1-P_{e}\right) \varepsilon+(\alpha+\xi) \Delta T\right] \lambda_{B}
$$

\section{DISCCUSION}

The development of new sensors will benefit from the advantages of FBG in CYTOP fiber reported in this paper. The refractive index of the CYTOP POF at $600 \mathrm{~nm}$ region is similar to the refractive index of water at $25^{\circ} \mathrm{C}$ [18] and such feature can be employed to detect oil and water layers in crude oil tanks [19]. Furthermore, this polymer is more chemically stable against chemical solutions and has a lower absorption of water than PMMA and other amorphous transparent thermoplastics [20]. These advantages make CYTOP POF sensor as a promising sensing technology in medical industry and also, as biosensors for compounds detection in aqueous solutions. Although CYTOP fibers present low optical losses at $1550 \mathrm{~nm}$ region, it generally needs a connectorization with silica fibers, which leads to further optical losses (see Fig. 4). Moreover, the butt-coupling process is very sensitive to misalignments that results in large spectral changes in the reflection spectrum as shown in Fig. 3. However, this is not a real limitation in applications using the visible wavelength range where low-cost components such as light sources, couplers and spectrometers are employed, and there is no need of butt-coupling between silica and POFs.

Visible light communications (VLC) also offer promising applications for FBGs in CYTOP POF, where they can be employed for signal routing and broadband optical source slicing [21]. 
Moreover, a FBG array in the visible wavelength range can also be used to generate different wavelengths from a single white light source [22] and color-shift keying (CSK) modulation can be implemented in VLCs [23].

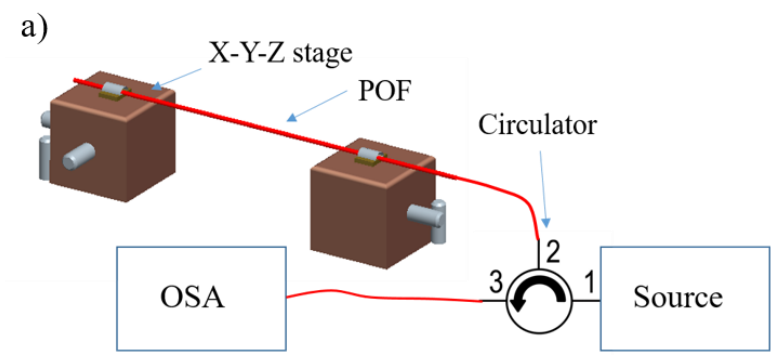

b)

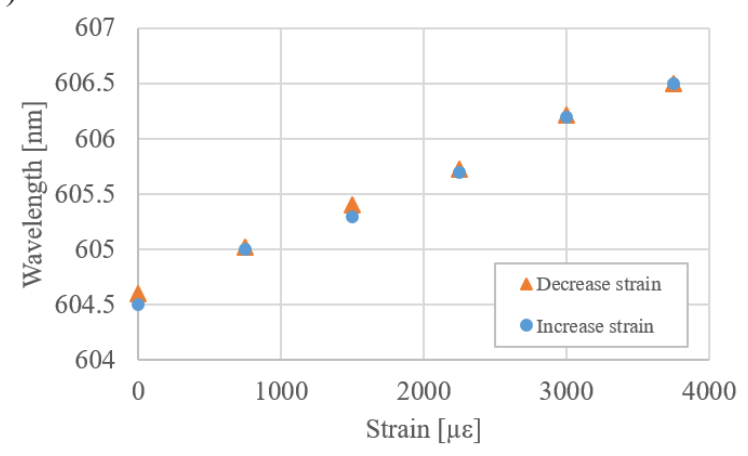

Fig. 6. (a) Strain setup used. (b) Wavelength shift under different strain.

\section{CONCLUSION}

In conclusion, we demonstrate the first FBG fabricated at $600 \mathrm{~nm}$ region in a commercial CYTOP POF using phase mask technology. This wavelength region is critical to be achieved by using fs laser systems due to the FBG period required. However, further efforts will be made in order to demonstrate stronger gratings. The temperature, humidity and strain characterization positively assess the use of these gratings for novel potential sensing and optical communication applications, which are also discussed. As future work we also plan to investigate the higher order modes' response to temperature and strain changes.

\section{ACKNOWLEDGMENT}

The authors acknowledge the financial support from FCT through the fellowship SFRH/BPD/109458/2015, program UID/EEA/50008/2013 by the National Fundsthrough the Fundação para a Ciência e a Tecnologia / Ministério da Educação e Ciência, and the European Regional Development Fund under the PT2020 Partnership Agreement. This work was also supported by KJCXZD201703 and the Research Excellence Award Programme GVA PROMETEO2017/103.

\section{REFERENCES}

[1] Y.Shi, E. Tangdiongga, A. M. J. Koonen, A. Bluschke, P. Rietzsch, J. Montalvo, M. Laat, G. Hoven, and B. Huiszoon. (2014) "Plastic-optical-fiber-based in-home optical networks.” IEEE Communications Magazine vol.52, no.6, pp.186-193.
[2] D. Sáez-Rodríguez, K. Nielsen, H. K. Rasmussen, O. Bang, D. J. Webb. (2013) "Highly photosensitive poly methy lmethacry late microstructured polymer optical fiber with doped core." Opt. Letters, vol.38, no.19, pp.3769-3772.

[3] X. Hu, C. F. J. Pun, H. Y. Tam, P. Mégret, and C. Caucheteur (2014) "Highly reflective Bragg gratings in slightly etched step-index polymer optical fiber," Opt. Express, vol.22, no.15, pp.18807-18817.

[4] X.Hu, G. Woyessa, D. Kinet, J. Janting, K. Nielsen, O. Bang, C. Caucheteur.(2017) "BDK-doped core microstructured PMMA optical fiber for effective Bragg grating photo-inscription." Opt. Letters, vol.42, no.11, pp.2209-2212.

[5] J. Bonefacino, H.-Yaw Tam, T. S. Glen, X. Cheng, C. -F. Jeff Pun, J. Wang, P. Heng Lee, M. -L. V. Tse, S. T. Boles. (2018) "Ultra-fast polymer optical fibre Bragg grating inscription for medical devices.” Light: Science \& Applications, vol.7, no.3, 17161.

[6] A. Lacraz, M. Polis, A. Theodosiou, C. Koutsides, K. Kalli, (2015) "Femtosecond laser inscribed Bragg gratings in low loss CYTOP polymer optical fiber." IEEE Photon. Technol. Lett., vol. 27, no.7, pp.693-696.

[7] M. Koerdt, S. Kibben, J. Hesselbach, C. Brauner, A. S.Herrmann, F.Vollertsen, , L. Kroll,(2014) "Fabrication and characterization of Bragg gratings in a graded-index perfluorinated polymer optical fiber." Procedia Technology, vol.15, pp.138-146.

[8] R. Olshansky. (1979) "Propagation in glass optical waveguides." Reviews of Modern Physics, vol.51, no.2, 341.

[9] C. Florea, K. A. Winick, (2003) "Fabrication and characterization of photonicdevices directly written in glass using femtosecond laser pulses." J. Lightwave Technol., vol.21, no.1, pp.246-253.

[10] R. Ishikawa, H. Lee, A. Lacraz, A. Theodosiou, K. Kalli, Y. Mizuno, K. Nakamura. (2017) "Pressure Dependence of Fiber Bragg grating inscribed in perfluorinated poly mer fiber." IEEE Photon. Technol. Lett., vol.29, no.24, pp.2167-2170.

[11] A. Theodosiou, A. Lacraz, M. Polis, K. Kalli, M. Tsangari, A. Stassis, M. Komodromos.(2016) "Modified fs-laser inscribed FBG array for rapid mode shape capture of free-free vibrating beams.” IEEE Photon. Technol. Lett., vol.28, no.14, pp.1509-1512.

[12] D. Vilarinho, A. Theodosiou, C. Leitão, A. G. Leal-Junior, M. D. F., Domingues, K. Kalli, Paulo Andre, Paulo Antunes, C. Marques. (2017) "POFBG-Embedded Cork Insole for Plantar Pressure M onitoring.” Sensors, vol.17, no.12, 2924.

[13] A. Theodosiou, M. Komodromos, K. Kalli. (2018) "Carbon Cantilever Beam Health Inspection Using a Polymer Fiber Bragg Grating Array.”J. Lightwave Technol., vol.36, no.4, pp.986-992

[14] K. Toma, Y. Masaki, M. Kusaba, K. Hirosawa, F. Kannari. (2015) “Control of grating-coupled ultrafast surface plasmon pulse and its nonlinear emission by shaping femtosecond laser pulse", Journal of Applied Physics, vol.118, no.10, 103102.

[15] Thorlabs, “Graded-Index Polymer Optical Fiber (GI-POF).” [Online]. Available: https://www.thorlabs.com/catalogPages/1100.pdf. [Conferred on: 28-April-2018].

[16] A. Pospori, C. A. F.Marques, D. Sáez-Rodríguez, K. Nielsen, O. Bang, D. J. Webb. (2017) "Thermal and chemical treatment of polymer optical fiber Bragg grating sensors for enhanced mechanical sensitivity." Opt. Fiber Technol., vol.36, pp. 68-74.

[17] B. Ortega, R. Min, D. Sáez-Rodríguez, Y. Mi, K. Nielsen, O. Bang, (2017) "Bandpass transmission filters based on phase shifted fiber Bragg gratings in microstructured polymer op tical fibers." SPIE Europe Vol. 10232, p. 1023209.

[18] R. Gravina, G. Testa, R. Bernini. "Perfluorinated plastic optical fiber tapers for evanescent wave sensing." Sensors, 9: 10423-33, 2009.

[19] A. G. Leal-Junior, C. Marques, A. Frizera, and M. J. Pontes, (2018) "Multi-interface level in oil tanks and applications of optical fiber sensors," Opt. Fiber Technol., vol.40, pp.82-92.

[20] M-H. Hung, P. R. Resnick, B. E. Smart, W. H. Buck. Fluorinated plastics, amorphous. In: Salamone JC, editor. Concise polymeric materials encyclopedia, Boca Raton: CRC Press; pp. 499-501, 1999.

[21] M. H. Reeve, A. R. Hunwicks, W. Zhao, S. G. Methley, L. Bickers, and S. Hornung, (1988) “LED spectral slicing for single-mode local loop applications," Electron. Lett., vol. 24 , no. 7 , p. 389.

[22] L. U. Khan, (2017) "Visible light communication: Applications, architecture, standardization and research challenges," Digit. Commun. Networks, vol. 3, no. 2, pp. 78-88, 2017.

[23] E. Monteiro and S. Hranilovic, (2014) "Design and Implementation of Color-Shift Key ing for Visible Light Communications," J. Light. Technol., vol. 32, no. 10, pp. 2053-2060. 